\title{
AC Losses and Their Thermal Effect in High-Temperature Superconducting Machines
}

\author{
Song, Xiaowei (Andy); Mijatovic, Nenad; Zou, Shengnan; Jensen, Bogi Bech; Holbøll, Joachim
}

Published in:

IEEE Transactions on Applied Superconductivity

Link to article, DOI:

10.1109/TASC.2016.2529293

Publication date:

2016

Document Version

Peer reviewed version

Link back to DTU Orbit

Citation (APA):

Song, X., Mijatovic, N., Zou, S., Jensen, B. B., \& Holbøll, J. (2016). AC Losses and Their Thermal Effect in HighTemperature Superconducting Machines. IEEE Transactions on Applied Superconductivity, 26(4), [5900105]. https://doi.org/10.1109/TASC.2016.2529293

\section{General rights}

Copyright and moral rights for the publications made accessible in the public portal are retained by the authors and/or other copyright owners and it is a condition of accessing publications that users recognise and abide by the legal requirements associated with these rights.

- Users may download and print one copy of any publication from the public portal for the purpose of private study or research.

- You may not further distribute the material or use it for any profit-making activity or commercial gain

- You may freely distribute the URL identifying the publication in the public portal 


\title{
AC Losses and Their Thermal Effect in High-Temperature Superconducting Machines
}

\author{
Xiaowei Song, Nenad Mijatovic, Member, IEEE, Shengnan Zou, Bogi Bech Jensen, Senior Member, IEEE, \\ and Joachim Holbøll, Senior Member, IEEE
}

\begin{abstract}
In transient operations or fault conditions, hightemperature superconducting (HTS) machines suffer ac losses, which have an influence on the thermal stability of superconducting windings. In this paper, a method to calculate ac losses and their thermal effect in HTS machines is presented. The method consists of three submodels that are coupled only in one direction. The magnetic field distribution is first solved in a machine model, assuming a uniform current distribution in HTS windings. The magnetic fields on the boundaries are then used as inputs for an ac loss model that has a homogeneous approximation and solves the $H$ formulation. Afterward, the computed ac losses are considered as the heat source in a thermal model to study the temperature profile in HTS windings. The method proposed is able to evaluate ac losses and their thermal effect, thus providing a reference to design an HTS machine and its cooling system.
\end{abstract}

Index Terms-AC losses, finite element methods, homogenization, superconducting machines, thermal stability.

\section{INTRODUCTION}

$\mathbf{H}$ IGH temperature superconducting (HTS) machines possess characteristics such as high power density and high efficiency, and they are preferable in applications such as ship propulsion and wind generators [1], [2]. Particularly, since direct drive wind generators with large power ratings will reduce the cost of wind farms, designs of HTS wind generators in $10 \mathrm{MW}$ rating and above have been of great interest in recent years [3]-[5].

There are many design proposals for HTS machines, most of which are based on partially superconducting machines, i.e., a copper AC armature winding and an HTS DC field winding [6]. Such a topology avoids large AC losses in HTS field windings. However, in transient operations or fault conditions HTS field windings still suffer AC losses. So the calculation of $\mathrm{AC}$ losses is of great significance to evaluate the performance of an HTS machine. Moreover, since AC losses are directly linked to the cost of a cooling system and the overall efficiency,

Manuscript received October 19, 2015; accepted February 3, 2016. Date of publication February 12, 2016; date of current version March 8, 2016.

X. Song, N. Mijatovic, and J. Holbøll are with the Center for Electric Power and Energy, Department of Electrical Engineering, Technical University of Denmark (DTU), 2800 Kongens Lyngby, Denmark (e-mail: song@elektro. dtu.dk).

S. Zou is with the Institute for Technical Physics, Karlsruhe Institute of Technology, 76344 Eggenstein-Leopoldshafen, Germany.

B. B. Jensen is with the Department of Science and Technology, University of the Faroe Islands, 100 Tórshavn, Faroe Islands.

Color versions of one or more of the figures in this paper are available online at http://ieeexplore.ieee.org.

Digital Object Identifier 10.1109/TASC.2016.2529293 careful consideration should be given to keep HTS machines competitive compared to their conventional counterparts.

Many methods and techniques have been reported to calculate AC losses [7]. Among these, the homogenization technique has been proven to be effective and it shows great advantages in simplicity and computation speed [8]-[10]. Nevertheless, very few works addressing AC losses have taken into account real operating conditions and constraints of an HTS machine.

In [11], a segregated model to calculate AC losses in an HTS generator was introduced. The method originally proposed in [11] is extended in this paper to study the excitation stage of a 2 MW HTS machine operating at $30 \mathrm{~K}$. First, an electromagnetic (EM) simulation towards the HTS machine is carried out, assuming a uniform current distribution in HTS windings. Subsequently, the magnetic fields encircling the HTS windings are extracted from the machine simulation results and used as boundary conditions in an AC loss model. The AC loss model in this paper has a homogenization approximation and solves the $\mathrm{H}$ formulation. Afterward, the calculated AC losses are considered as the heat source in a thermal model to study the temperature increase due to the AC losses. The method presented consists of three sub-models that couple only in one direction, and it is easy to implement. The results will benefit the design of an HTS machine and its cooling system.

\section{Modeling Strategy}

The method consists of three sub-models: a machine model, an AC loss model and a thermal model. Fig. 1 shows the flow of the modeling process.

First, an EM simulation of the HTS machine is carried out, assuming a uniform current distribution in the HTS windings. Disregarding the actual current distribution in superconducting windings has a negligible influence on the overall magnetic field profile [11]. Actually, this is a common principle used for EM design of HTS machines.

Subsequently, the magnetic fields encircling the HTS windings are extracted from the previous EM simulation, and these values are input as boundary conditions in an AC loss model. The AC loss model considers the $E-J$ power law, an important superconducting property, so that the actual current distribution and AC losses can be determined.

Finally, the AC losses calculated are considered as the heat source in HTS windings to calculate the temperature increase due to the dissipation of these $\mathrm{AC}$ losses. If the temperature increase is considerable, then the critical current density (noted as $J_{c}$ ) in the AC loss model needs to be modified, and an 


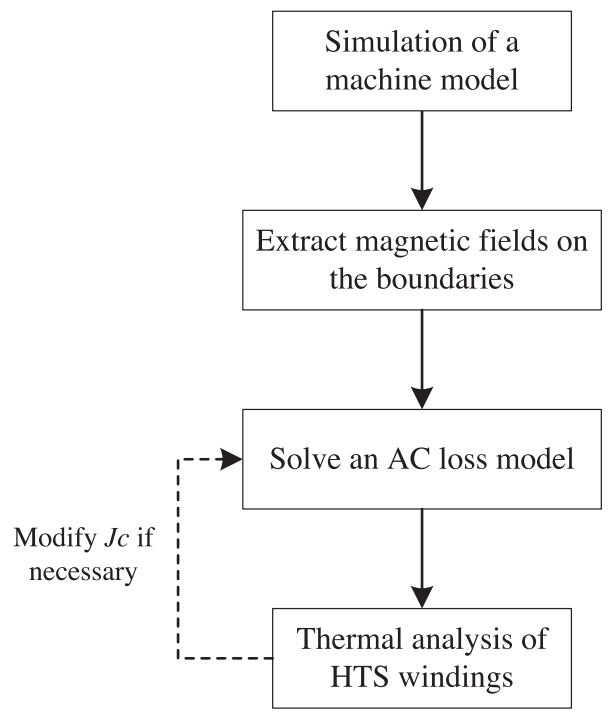

Fig. 1. Flowchart of the modeling strategy to compute ac losses and their thermal effect in an HTS machine.

TABLE I

DESIGN SPECIFICATIONS OF A DD HTS WIND GENERATOR

\begin{tabular}{ll}
\hline \hline Rated output (MW) & 2 \\
Pole pair number & 12 \\
Rated rotating speed (rpm) & 10 \\
Outer diameter (m) & 3.96 \\
Axial length (m) & 1 \\
Rated current in HTS windings (A) & 480 \\
Operating temperature of HTS windings (K) & 30 \\
Number of layers per HTS winding & 2 \\
Number of HTS tapes per layer & 250 \\
\hline \hline
\end{tabular}

iterative computation is required. For the case considered in this paper, since the temperature increase is smaller than $0.5 \mathrm{~K}$, the modification of $J_{c}$ is not performed.

\section{DESCRIPTION OF Sub-MODEls}

\section{A. Machine Model}

A 2 MW direct drive (DD) HTS wind generator is studied here. The design specifications are listed in Table I and the schematic configuration for a pole pair segment is illustrated in Fig. 2.

The generator considered employs HTS windings in the rotor and copper windings in the stator. Comparative studies have shown that the topology with a salient-pole iron rotor and an iron toothed stator, which is also the one used here, is the most cost-effective option given the current price of HTS tapes commercially available in the market [12]. An iron pole is placed in the center of every HTS winding, and is separated from the HTS winding by $10 \mathrm{~mm}$, so that the magnetic flux will mainly go through the iron pole, instead of the HTS winding. The losses experienced by the iron pole are neglected in this paper, which could be supported from two aspects. One is that the iron pole is highly saturated, resulting in minor hysteresis loops, and only a small portion of the iron pole is affected by AC flux ripples and harmonics from the stator. Minor hysteresis

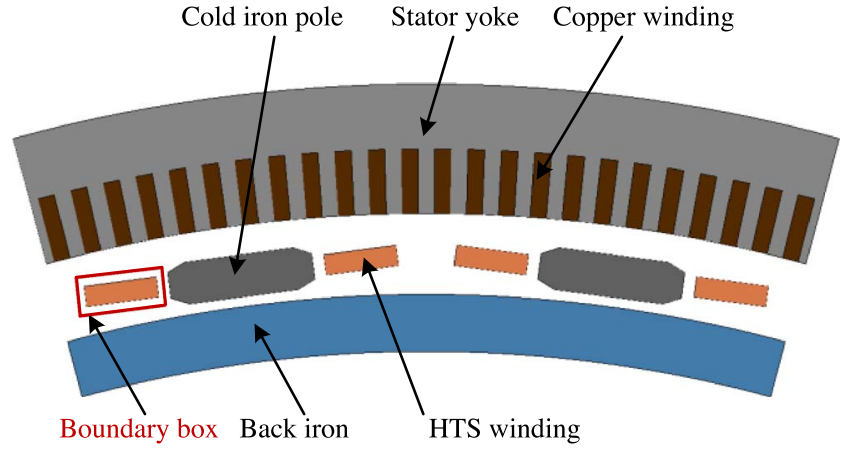

Fig. 2. Illustration of a pole pair segment in a 2-MW partially HTS wind generator. The red rectangle represents the coupling boundary of the machine model and the ac loss model.

loops coupled with a small amount of material cause low hysteresis losses. The other is that a low frequency gives rise to low eddy current losses, even if eddy current losses are proved to be an issue, laminated steel can be implemented to reduce those.

\section{B. AC Loss Model}

The AC loss model solves the $\mathrm{H}$ formulation, which is written as [10], [13], [14]:

$$
\nabla \times(\rho \nabla \times \mathbf{H})+\frac{\partial(\mu \mathbf{H})}{\partial t}=0
$$

where $\rho, \mu$, and $\mathbf{H}$ are the resistivity, permeability and the magnetic field, respectively. In this paper, the ferromagnetic losses due to the magnetic substrate in HTS tapes are not considered, so $\mu=\mu_{0}$, which is the air permeability, holds throughout the paper. The resistivity of normal conductors can be treated as constant, while for the HTS material, the $E-J$ power law is used to describe the non-linear transition from the superconducting state to the normal state:

$$
\rho_{\mathrm{sc}}=\frac{E_{c}}{J_{c}}\left(\frac{J}{J_{c}}\right)^{n-1}
$$

where $E_{c}=1 \mu \mathrm{V} / \mathrm{cm}$ and $n$ (assumed as 40 in this paper) is the index describing how abruptly the transition takes place. $J$ represents the current density.

$J_{c}$ is both field dependent and temperature dependent. The field dependence of $J_{c}$ at the operating temperature $(30 \mathrm{~K})$ is elaborated in Fig. 3. ${ }^{1}$ As will be seen later, the temperature increase due to the $\mathrm{AC}$ losses for the case studied in this paper is very limited, its influence on the critical current density is negligible, so the temperature dependence of $J_{c}$ is not considered.

The homogenization technique introduced in [8]-[10] is used to compute AC losses. The advantage of this technique is that it disregards the inner details of an HTS winding, then the HTS winding can be studied as a homogenized bulk, giving rise to a faster computation without compromising the accuracy.

The red rectangle shown in Fig. 2 denotes the coupling boundary of the machine model and the AC loss model. The

\footnotetext{
${ }^{1}$ Technical Documents Provided by AMSC
} 


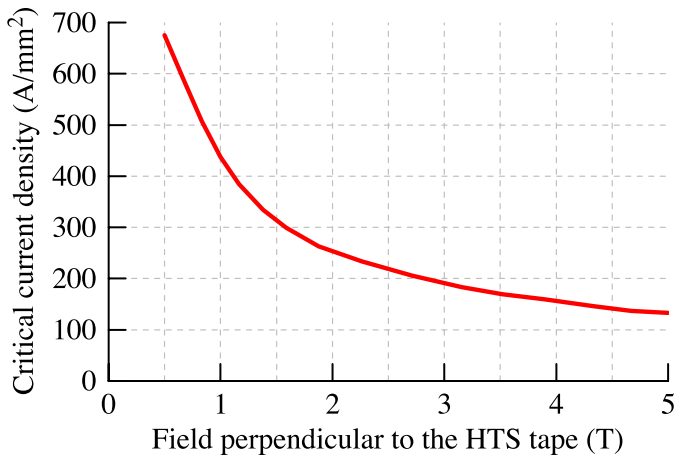

Fig. 3. Field dependence of the critical current density for AMSC's Amperium 12-mm HTS tape operating at $30 \mathrm{~K}$. The tape has a critical current of $350 \mathrm{~A}$ at $77 \mathrm{~K}$, self-field.

magnetic fields and their variations along the lines in the red rectangular are extracted from the machine simulation results and applied as boundary conditions in the AC loss model, so that the machine parameters are not necessary in the AC loss model. This simplifies the problem greatly. Because there are many machine parameters that influence the AC loss computation, e.g., geometric dimensions, non-linear magnetic properties of iron components, load variations in the armature, directly considering a full-fledged machine model makes the AC loss computation cumbersome.

It is necessary to point out that the technique of using the coupling boundary to simplify the problem can be extended. In Fig. 2, the coupling boundary encircles a complete HTS field winding. However, if the HTS field winding is very large, splitting the coupling boundary around a complete HTS winding results in a large $\mathrm{AC}$ loss computing domain, which makes the AC loss simulation computationally demanding. Under these circumstances, the coupling boundary can just encircle a small portion of the HTS winding, e.g. one quarter portion or even smaller. The magnetic field will be evaluated individually on the coupling boundary in every small portion, and then applied to its corresponding $\mathrm{AC}$ loss computing domain.

\section{Thermal Model}

The thermal model is based on the time-dependent heat transfer equations:

$$
\begin{aligned}
C_{v} \frac{\partial T}{\partial t} & =\nabla \cdot(\lambda \nabla T)+Q_{\mathrm{ac}} \\
Q_{\mathrm{ac}} & =E J
\end{aligned}
$$

where $T$ is the temperature; $C_{v}$ and $\lambda$ are the volumetric heat capacity and the thermal conductivity, respectively. $Q_{\mathrm{ac}}$ is the heat power density generated by the AC losses in the HTS winding, which equals the product of the electric field $E$ and the current density $J$.

The equivalent thermal properties of the HTS winding are composites of the HTS tape and the epoxy. The volumetric heat capacity of the HTS winding is written as:

$$
C_{v_{-} \text {winding }}=C_{v_{-} \text {tape }} f_{\text {tape }}+C_{v_{-} \text {epoxy }}\left(1-f_{\text {tape }}\right)
$$

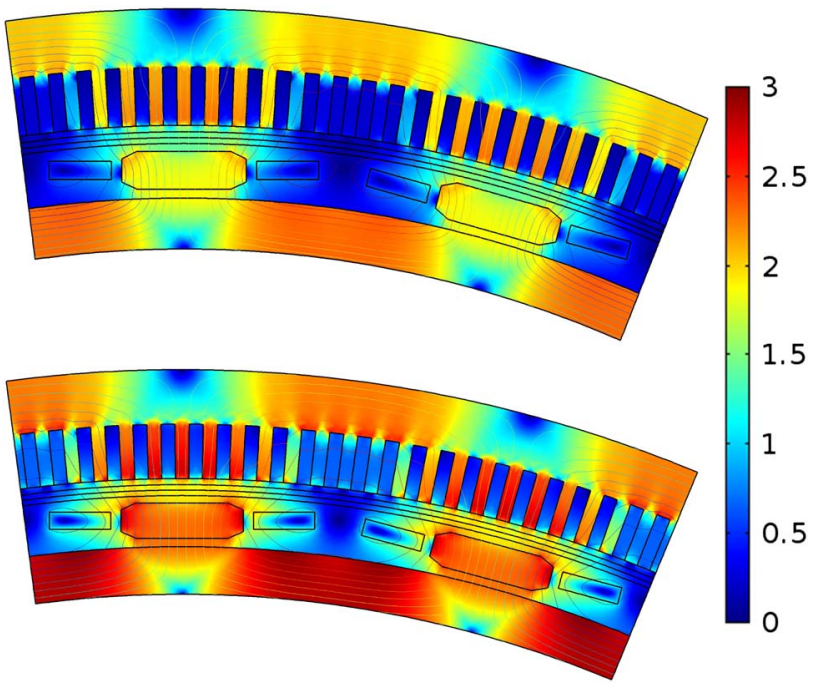

Fig. 4. The magnetic field distributions in the machine at $t=60 \mathrm{~s}$ (upper) and $t=120 \mathrm{~s}$ (lower). The current in the HTS winding is ramped up from 0 to $480 \mathrm{~A}$ at a constant rate of $4 \mathrm{~A} / \mathrm{s}$.

where $f_{\text {tape }}$ is the volumetric fraction of the HTS tape in the winding. The thermal conductivity of the HTS winding is anisotropic, which is expressed as:

$$
\begin{aligned}
& \lambda_{x_{-} \text {winding }}=\frac{\lambda_{\text {tape }} \cdot \lambda_{\text {epoxy }}}{\lambda_{\text {tape }}\left(1-f_{\text {tape }}\right)+\lambda_{\text {epoxy }} f_{\text {tape }}} \\
& \lambda_{y_{-} \text {winding }}=\lambda_{\text {tape }} f_{\text {tape }}+\lambda_{\text {epoxy }}\left(1-f_{\text {tape }}\right)
\end{aligned}
$$

where $\lambda_{x_{-} \text {winding }}$ and $\lambda_{y_{-} \text {winding }}$ are the $x$ component and $y$ component thermal conductivities of the HTS winding, respectively. The thermal properties of different materials at $30 \mathrm{~K}$ used in the model can be found in [15], [16]. ${ }^{2}$

The boundary condition is assigned as thermal insulation with an assumption that all other heat loads, e.g., radiation heat load and conduction heat load, are balanced with the cooling power of the cryocooler. So the temperature increase is due solely to the AC losses.

\section{RESUlTS}

\section{A. Machine Simulation}

An EM simulation of a pole pair segment of the proposed machine is carried out in COMSOL. A typical excitation stage of the HTS field winding is simulated. The current in the HTS winding is ramped up from 0 to $480 \mathrm{~A}$ at a constant rate of $4 \mathrm{~A} / \mathrm{s}$. Fig. 4 shows the magnetic field distributions when the time is $60 \mathrm{~s}$ and $120 \mathrm{~s}$, respectively. As can be seen, the magnetic field experienced by the HTS winding is varying with respect to the time, especially the upper-right part of the HTS winding, which faces the stator and iron pole. Due to the symmetry, only the magnetic field encircling half of an HTS winding needs to be assessed for the AC loss calculation. To easily assess the magnetic fields by the coordinates of the points

\footnotetext{
htm.
} 


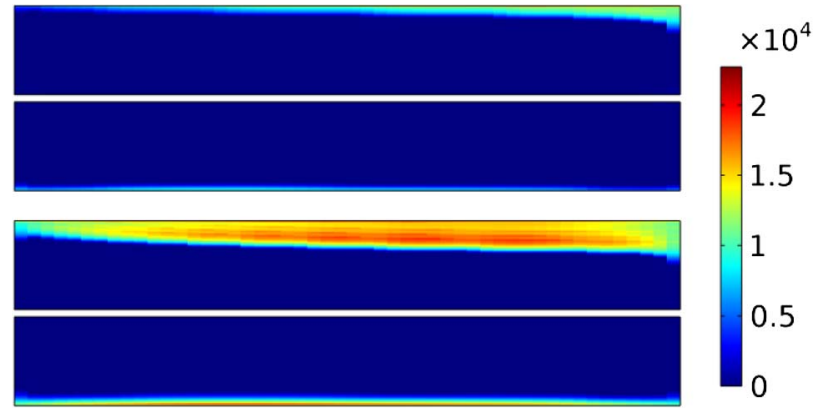

Fig. 5. The distributions of the ac loss density $Q_{\mathrm{ac}}\left(\mathrm{W} / \mathrm{m}^{3}\right)$ for half of an HTS winding at $t=60 \mathrm{~s}$ (upper) and $t=120 \mathrm{~s}$ (lower).

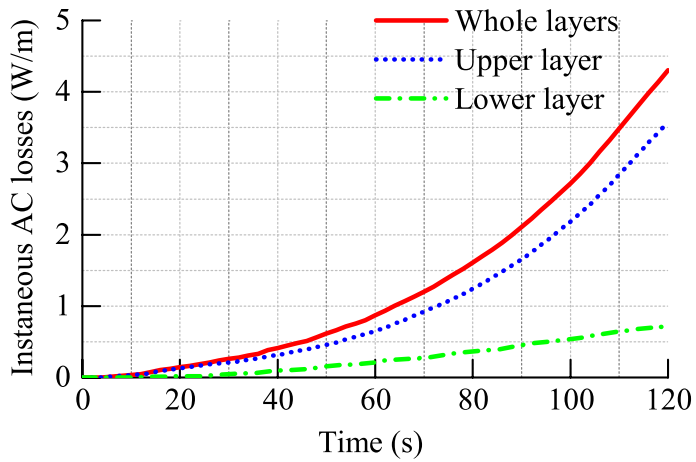

Fig. 6. Instantaneous ac losses for the upper HTS layer, lower HTS layer, and the whole layers. Note that only half of an HTS winding is considered; hence, the values shown here should be doubled to evaluate the actual ac losses in a full HTS winding.

on the coupling boundary, the left HTS winding in Fig. 4 is deliberately placed in parallel with the global coordinate system.

\section{B. AC Losses}

The AC losses generated in the HTS field winding during the excitation stage is calculated by the aforementioned AC loss model. The distributions of the AC loss density $Q_{\mathrm{ac}}$ and the instantaneous AC losses for half of an HTS winding are shown in Figs. 5 and 6, respectively. As indicated in Figs. 5 and 6, the upper HTS layer, which faces a higher flux density due to the stator, has higher AC losses. It also can be seen that the closer to the iron pole, the higher loss density will be. The effect of the inhomogeneous distribution of the AC loss density will be displayed explicitly in the temperature profile.

\section{Thermal Simulation}

The AC loss density obtained in the above section is input as a heat source in the thermal model to evaluate the temperature increase. The temperature distributions for half of an HTS winding at $t=60 \mathrm{~s}, t=90 \mathrm{~s}$, and $t=120 \mathrm{~s}$ are shown in Fig. 7 . The temperature starts increasing at the upper-right corner and gradually penetrates towards the interior, which is consistent with the loss density distribution shown in Fig. 5. As a result, a maximum temperature rise of $0.4 \mathrm{~K}$ happens at the upperright corner, and a minimum value of $0.12 \mathrm{~K}$ is observed at the lower-left corner, as marked in Fig. 7. The temperature profile

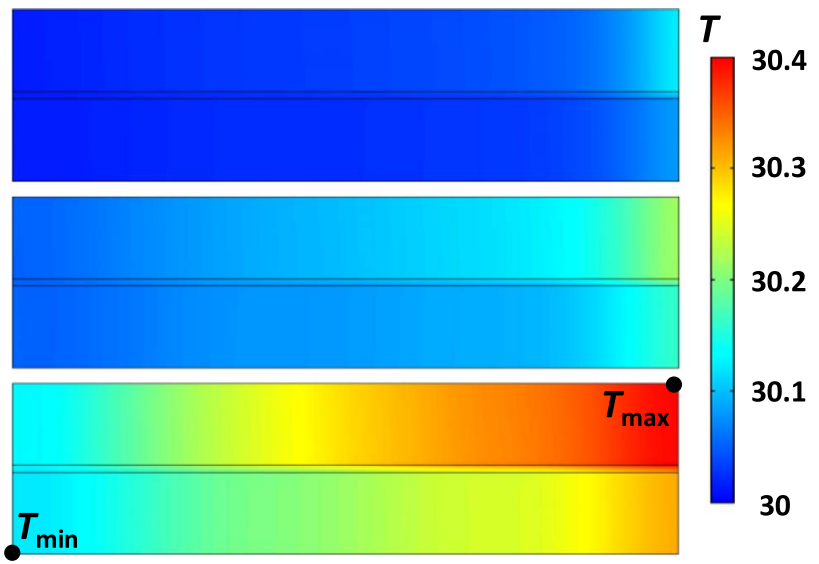

Fig. 7. The temperature distribution for half of an HTS winding at $t=60 \mathrm{~s}$ (upper), $t=90 \mathrm{~s}$ (middle), and $t=120 \mathrm{~s}$ (lower). $T_{\max }$ and $T_{\min }$ are the points that have the maximum and minimum temperature rise, respectively.

obtained can serve as a reference to design the cooling system. Since the temperature increase evaluated has a limited influence on $J_{c}$ (less than 5\%), the modification of $J_{c}$ is not carried out.

\section{CONCLUSion}

Three sub-models are considered together to calculate AC losses and their thermal effect in an HTS machine. The aforementioned machine model and AC loss model are only linked on the coupling boundary, thus the machine parameters are not necessary in the AC loss computation. The AC loss density calculated in the AC loss model is input as a heat source to study the thermal profile of the HTS winding. In the case studied, the $\mathrm{AC}$ losses generated during the excitation stage cause a maximum temperature rise of $0.4 \mathrm{~K}$ at the corner that faces the stator and iron pole. The method proposed provides a reference to evaluate an HTS machine and its cooling system.

The AC loss due to the variations of the armature load and the rotating speed will be addressed in our future work.

\section{ACKNOWLEDGMENT}

The authors would like to thank Dong Liu, a Ph.D. student in the Department of Electrical Sustainable Energy, Delft University of Technology, Netherlands, for his troubleshooting in COMSOL and technical discussion as well.

\section{REFERENCES}

[1] B. B. Jensen, N. Mijatovic, and A. B. Abrahamsen, "Development of superconducting wind turbine generators," J. Renew. Sustain. Energy, vol. 5, no. 2, Mar. 2013, Art. ID 023137.

[2] J. Voccio et al., "2G HTS wires and the implications for motor and generator applications," IEEE Trans. Appl. Supercond., vol. 17, no. 2, pp. 1591-1594, Jun. 2007.

[3] A. B. Abrahamsen et al., "Superconducting wind turbine generators," Supercond. Sci. Technol., vol. 23, no. 3, 2010, Art. ID 034019.

[4] G. Snitchler, B. Gamble, C. King, and P. Winn, "10 MW class superconductor wind turbine generators," IEEE Trans. Appl. Supercond., vol. 21, no. 3, pp. 1089-1092, Jun. 2011.

[5] Y. Terao, M. Sekino, and H. Ohsaki, "Comparison of conventional and superconducting generator concepts for offshore wind turbines," IEEE Trans. Appl. Supercond., vol. 23, no. 3, Jun. 2013, Art. ID 5200904. 
[6] X. Song, N. Mijatovic, B. B. Jensen, and J. Holboll, "Design study of fully superconducting wind turbine generators," IEEE Trans. Appl. Supercond., vol. 25, no. 3, Jun. 2015, Art. ID 5203605.

[7] F. Grilli et al., "Computation of losses in HTS under the action of varying magnetic fields and currents," IEEE Trans. Appl. Supercond., vol. 24, no. 1, Feb. 2014, Art. ID 8200433.

[8] J. R. Clem, J. Claassen, and Y. Mawatari, "AC losses in a finite Z stack using an anisotropic homogeneous-medium approximation," Supercond. Sci. Technol., vol. 20, no. 12, pp. 1130-1139, 2007.

[9] W. Yuan, A. Campbell, and T. Coombs, "A model for calculating the AC losses of second-generation high temperature superconductor pancake coils," Supercond. Sci. Technol., vol. 22, no. 7, 2009, Art. ID 075028.

[10] V. M. Zermeno, A. B. Abrahamsen, N. Mijatovic, B. B. Jensen, and M. P. Sørensen, "Calculation of alternating current losses in stacks and coils made of second generation high temperature superconducting tapes for large scale applications," J. Appl. Phys., vol. 114, no. 17, 2013, Art. ID 173901.

[11] V. M. Zermeno et al., "Simulation of an HTS synchronous superconducting generator," Phys. Procedia, vol. 36, pp. 786-790, 2012.
[12] H. Karmaker, M. Ho, and D. Kulkarni, "Comparison between different design topologies for multi-megawatt direct drive wind generators using improved second generation high temperature superconductors," IEEE Trans. Appl. Supercond., vol. 25, no. 3, Jun. 2015, Art. ID 5201605.

[13] Z. Hong and T. A. Coombs, "Numerical modelling of AC loss in coated conductors by finite element software using H formulation," J. Supercond. Nov. Magn., vol. 23, no. 8, pp. 1551-1562, 2010.

[14] Z. Hong et al., "AC losses of superconducting racetrack coil in various magnetic conditions," IEEE Trans. Appl. Supercond., vol. 21, no. 3, pp. 2466-2469, Jun. 2011.

[15] H. Bai, W. D. Markiewicz, J. Lu, and H. W. Weijers, "Thermal conductivity test of YBCO coated conductor tape stacks interleaved with insulated stainless steel tapes," IEEE Trans. Appl. Supercond., vol. 23, no. 3, Jun. 2013, Art. ID 4600204.

[16] M. Bonura and C. Senatore, "High-field thermal transport properties of REBCO coated conductors," Supercond. Sci. Technol., vol. 28, no. 2, 2015, Art. ID 025001. 